\title{
Task-related brain activation during finger tapping and circle drawing monitored by fNIRS
}

\author{
Shuichi Matsuzaki, Masamichi Morihiro, Tadashi Tsubone, Yasuhiro Wada \\ From Nineteenth Annual Computational Neuroscience Meeting: CNS*2010 \\ San Antonio, TX, USA. 24-30 July 2010
}

The present study assessed brain activation measured by functional near-infrared spectroscopy (fNIRS) in which subjects perform two types of hand motor tasks: a finger tapping task and circle drawing task. For both experiments we employed 24-channel NIRS system covering the left hemisphere (Fig. 1(a)) The typical time course in oxygenated hemoglobin concentration $(o x y H b)$ and deoxygenated hemoglobin concentration (deoxyHb) are shown in Fig. 2. In finger-tapping tasks, four righthanded subjects performed right hand finger tapping at a rate of approx. $3 \mathrm{~Hz}$ paced by a metronome. Subjects performed 12 or 15 trials in which a trial consists of 10-s rest, 20-s finger tapping movement, 30 -s rest and about 180-s of inter-task intervals. Changes in oxyHb and deoxyHb were measured at a sampling period of $130-\mathrm{ms}$. Also changes in the angular velocity of finger tapping, as a measure of the movement smoothness, were measured. Significant decrease in oxyHb and the angular velocity over time were observed, while those transitions further exhibited significant correlation at the channels covering SMA, the pre-motor area (PMA) and PFC (Fig. 1(b)). In circle drawing tasks, six righthanded subjects (males from 22 to 24 years old) performed circle drawing with right hand. Subjects were instructed to trace around a template circle at the frequency of $0.667 \mathrm{~Hz}$. All subjects performed four trials

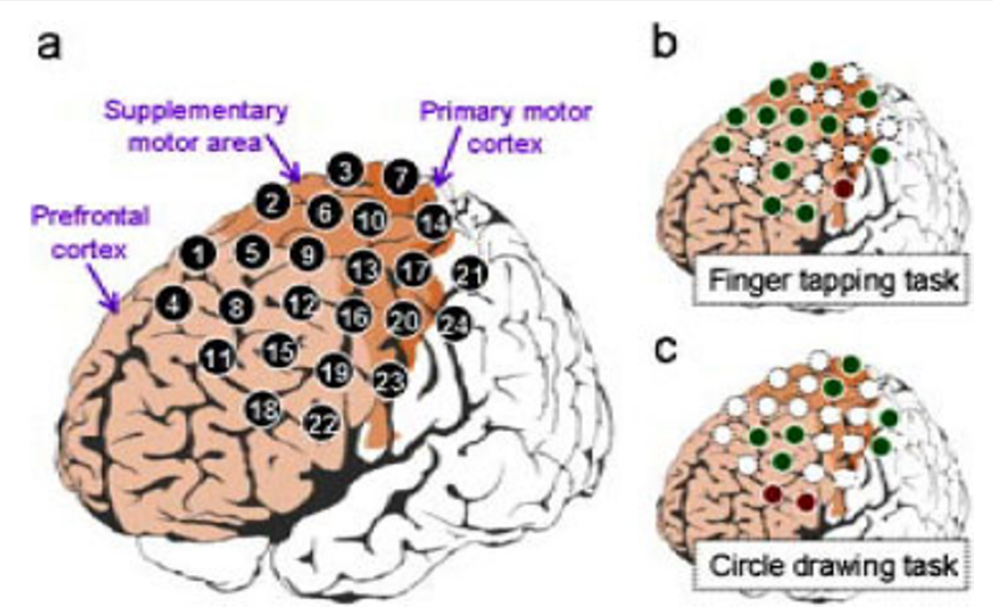

Figure 1 (a) Channel setups in fNIRS measurements. (b) Regional activations during finger tapping tasks. Colored area represents where changes in oxyHb were significantly correlated with the task-performance (i.e. tapping smoothness) in all subjects (red), all except one subject (green). (c) Regional activations during circle drawing tasks. Colored area represents where changes in oxyHb were correlated with the task performance (i.e. circle drawing deviations from a template) in all subjects (red), all except one subject (green).

\footnotetext{
* Correspondence: shmatsu@nagaokaut.ac.jp

Department of Electrical Engineering, Nagaoka University of Technology, Nagaoka, Japan
} 

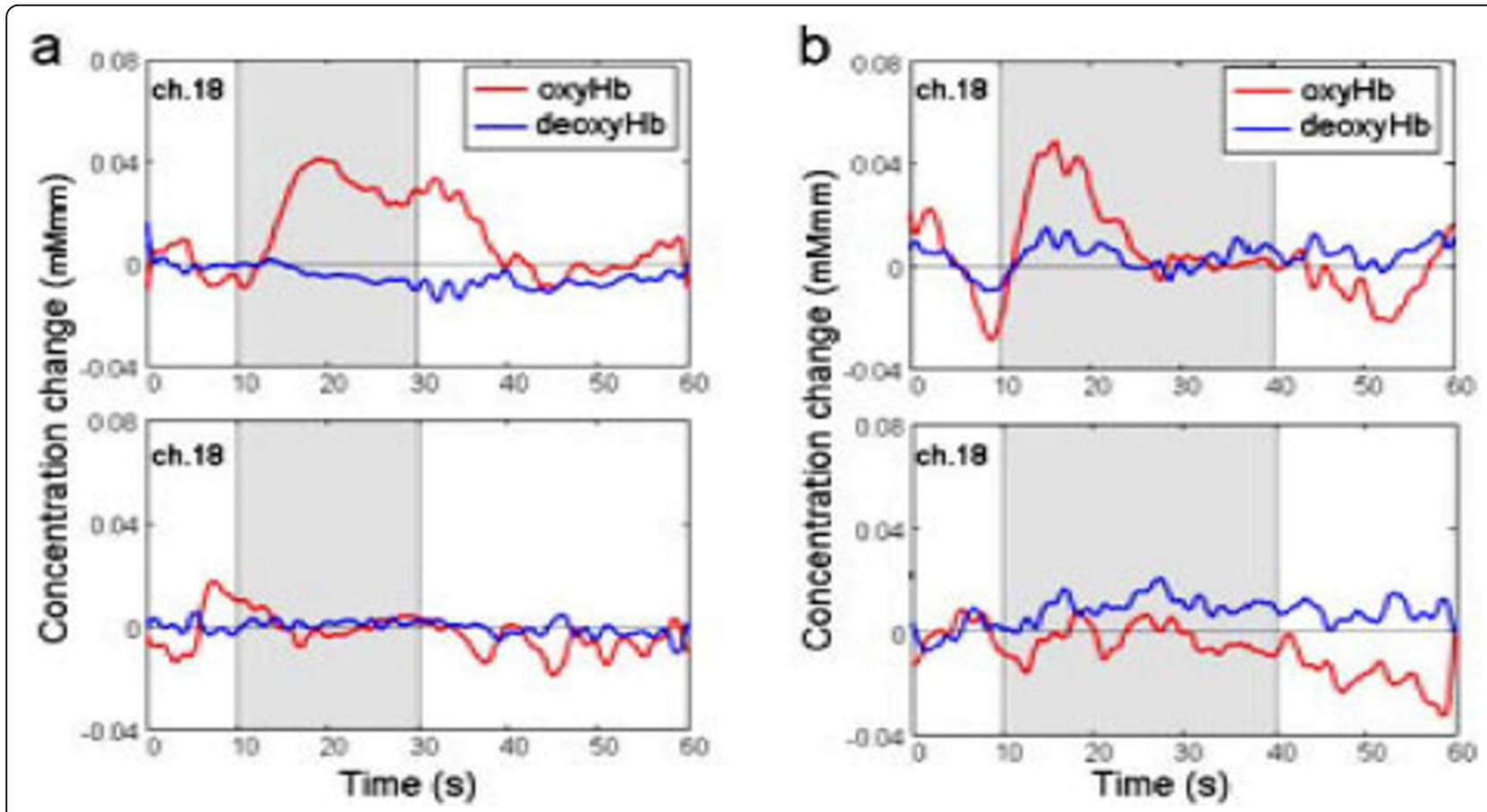

Figure $\mathbf{2}$ Typical time course of the hemoglobin concentration changes at channel $\mathbf{1 8}$ over the prefrontal cortex. (a) The result of finger tapping task at the first trial period (top) and the 15th trial period (bottom). (b) The result of circle drawing task at the first trial period (top) and the fourth trial period (bottom).

where each consists of 10-s rest, 30-s circle drawing, 30 -s rest and about 180 -s intervals. We calculated a spatial error as a measure of task performance which is derived from the trajectory deviated from the template circle. We found that oxyHb as well as error rate were decreased over time, with those transitions further be significantly correlated at the channels covering PFC and M1 (Figure 1(c)).

In summary, this paper reports our empirical results using the two types of hand motor tasks. The experimental results indicated the task-related activity observed in NIRS signals. A previous fNIRS study using hand motor tasks showed similar results including decreased pattern of hemodynamics in SMA [1], whereas further investigation will be desired that may allows to estimate precise cortical functions reflecting motor behaviors.

Published: 20 July 2010

\section{Reference}

1. Hatakenaka M, Miyai I, Mihara M, Sakoda S, Kubota K: Frontal regions involved in learning of motor skill -A functional NIRS study. Neurolmage 2007, 34:109-116.

\section{doi:10.1186/1471-2202-11-S1-P87}

Cite this article as: Matsuzaki et al:: Task-related brain activation during finger tapping and circle drawing monitored by fNIRS. BMC Neuroscience 2010 11(Suppl 1):P87.
Submit your next manuscript to BioMed Central and take full advantage of:

- Convenient online submission

- Thorough peer review

- No space constraints or color figure charges

- Immediate publication on acceptance

- Inclusion in PubMed, CAS, Scopus and Google Scholar

- Research which is freely available for redistribution 